\title{
HQEFT as A Large Component QCD and Comments on The Incompleteness of HQET
}

\author{
Y.L. Wu, Y.A. Yan, M. Zhong and Y.B. Zuo \\ Institute of Theoretical Physics, Chinese Academy of Sciences, \\ P.O. Box 2735, Beijing 100080, China \\ W. Y. Wang \\ Department of Physics, Tsinghua University, Beijing 100084, China
}

\begin{abstract}
The Heavy quark effective field theory (HQEFT) is revisited in a more intuitive way. It is shown that HQEFT is a consistent large component QCD of heavy quarks. In the non-relativistic limit, HQEFT recovers the non-relativistic QCD (NRQCD). The resulting new effects in the HQEFT of QCD are carefully reexamined. It is then natural to come to the comments on the usual heavy quark effective theory (HQET). Consistent phenomenological applications of HQEFT exhibit its interesting features and completeness in comparison with HQET. It then becomes manifest why we shall base on the HQEFT of QCD rather than HQET which is an incomplete one for computing $1 / m_{Q}$ corrections. More precise extraction for $\left|V_{c b}\right|$ and $\left|V_{u b}\right|$ in the HQEFT of QCD is emphasized.
\end{abstract}

PACS numbers: $12.39 . \mathrm{Hg}, 12.38$. Aw

Keywords: Effective theories of heavy quark, large component QCD, quark-antiquark couplings

\section{INTRODUCTION}

Two frameworks of effective theories for heavy quarks have extensively been applied to treat heavy quark systems containing a single heavy quark. One is so-called heavy quark effective theory (HQET) $[1,2]$ and the other is mentioned, for distinction, to be the heavy quark effective field theory (HQEFT) [3]. The former was motivated and constructed mainly based on the heavy quark symmetry (HQS) [4-6] in the infinity mass limit $m_{Q} \rightarrow \infty[7,8]$. The latter was derived directly from QCD with carefully treating the quark and anti-quark fields. In the HQET, the quark and antiquark fields were treated separately, which is valid only in the infinity mass limit. For the finite quark mass case, it is well known from the quantum field theory that the quark and antiquark fields cannot be separated though their interactions may be suppressed by the inverse of the mass. This is one of the main reasons that motivated one of our authors to consider and derive an alternative HQEFT from QCD with explicitly keeping the quark and antiquark coupling interactions [3]. In deriving the HQEFT, the approximate spin-flavor symmetry is not as the starting point but as a direct consequence in the heavy quark limit. In fact, it is the approximate spin-flavor symmetry that has led a rapid development on effective theories of heavy quark [9]- [19] in studying heavy quark systems. It is obvious that the two frameworks, HQET and HQEFT of $\mathrm{QCD}$, become equivalent only when taking the heavy quark mass to be infinite. This is because that only in this case the quark and antiquark can be completely decoupled. The possible differences between them have recently been investigated in detail from heavy hadron decays [20]- [29], where the important new effects have been found to arise from the $1 / m_{Q}$ corrections. The interesting applications of HQEFT have also been studied in the pair creations and annihilations [30-32]. In this paper, we are going to revisit the HQEFT of QCD in a more intuitive way and make it more clear for acceptance. Especially, one will see why HQEFT should be regarded as a more complete effective theory of QCD for heavy quark systems. To be manifest, we consider two interesting cases that can be dealt with by the HQEFT of QCD: one is for a heavy quark within the heavy quarkonia systems, which is known to be treated by the non-relativistic QCD (NRQCD) [33,34]; the other is for a single heavy quark within the heavy hadron systems [20]- [29]. Some comments on the HQET then become obvious. Several important and interesting observations yielded in the HQEFT of QCD are briefly summarized. The emphasis is made to the more precise extractions on the Cabbibo-Kobayashi-Maskawa (CKM) matrix elements $V_{c b}$ and $V_{u b}$ via HQEFT of QCD. 


\section{WHY HQEFT OF QCD RATHER THAN HQET}

HQEFT of QCD is a theoretical framework derived directly from QCD but explicitly displays the heavy quark symmetry and symmetry breaking corrections. The main point is that all contributions of the field components, large and small, 'particle' and 'antiparticle', have carefully been treated in the Lagrangian of HQEFT, so that the resulting effective Lagrangian form the basis for a "complete" effective field theory of heavy quarks [3,20-29]. We will see that HQEFT of QCD is actually an effective field theory of large component QCD.

Let us begin with the Lagrangian of full QCD

$$
\mathcal{L}_{Q C D}=\mathcal{L}_{\text {light }}+\bar{Q}\left(i \not D-m_{Q}\right) Q,
$$

where $Q$ is the quantum field for heavy quark and $\mathcal{L}_{\text {light }}$ represents the part that has nothing to do with the heavy quark. Following the derivation presented in ref. [3], the field $Q$ can formally be written into two part

$$
Q=Q^{(+)}+Q^{(-)}
$$

where $Q^{(+)}$and $Q^{(-)}$are classically corresponding to two solutions of Dirac equation

$$
\left(i \not D-m_{Q}\right) Q^{( \pm)}=0 .
$$

For the free quark fields, they are so called "quark" and "antiquark" fields respectively, and can be expanded in terms of plane waves

$$
\begin{aligned}
Q^{(+)}(x) & =\int \frac{d^{3} p}{(2 \pi)^{3}} \frac{m}{p^{0}} \sum_{s} b_{s}(p) u_{s}(p) e^{-i p \cdot x} \\
Q^{(-)}(x) & =\int \frac{d^{3} p}{(2 \pi)^{3}} \frac{m}{p^{0}} \sum_{s} d_{s}^{\dagger}(p) v_{s}(p) e^{i p \cdot x}
\end{aligned}
$$

where $s$ is spin index, $b_{s}$ and $d_{s}$ are the annihilation and creation operators respectively. $u_{s}$ and $v_{s}$ are four-component spinors. In Dirac representation, they can be explicitly written as

$$
\begin{aligned}
& u_{s}(p)=\sqrt{\frac{E+m}{2 m}}\left(\begin{array}{c}
1 \\
\frac{\sigma \cdot \mathbf{p}}{E+m}
\end{array}\right) \varphi_{s}, \\
& v_{s}(p)=\sqrt{\frac{E+m}{2 m}}\left(\begin{array}{c}
\frac{\sigma \cdot \mathbf{p}}{E+m} \\
1
\end{array}\right) \chi_{s}
\end{aligned}
$$

with $\varphi_{s}$ being the two component Pauli spinor field that annihilates a heavy quark, and $\chi_{s}$ being the Pauli spinor field that creates a heavy antiquark.

Introducing an arbitrary unit vector $v^{\mu}$ which satisfies $v^{2}=1$, we can further decompose the quark fields $Q^{( \pm)}$into the following forms

$$
\begin{aligned}
& Q^{(+)} \equiv\left(\frac{1+\psi}{2}+\frac{1-\psi}{2}\right) Q^{(+)}=\hat{Q}_{v}^{(+)}+R_{v}^{(+)}, \\
& Q^{(-)} \equiv\left(\frac{1-\psi}{2}+\frac{1+\psi}{2}\right) Q^{(-)}=\hat{Q}_{v}^{(-)}+R_{v}^{(-)}
\end{aligned}
$$

with

$$
\hat{Q}_{v}^{( \pm)} \equiv \frac{1 \pm \psi}{2} Q^{( \pm)}, \quad R_{v}^{( \pm)} \equiv \frac{1 \mp \psi}{2} Q^{( \pm)} .
$$

Thus the initial quark field $Q$ can be written as

$$
Q \equiv \hat{Q}_{v}+R_{v}
$$


with

$$
\hat{Q}_{v}=\hat{Q}_{v}^{(+)}+\hat{Q}_{v}^{(-)}, \quad R_{v}=R_{v}^{(+)}+R_{v}^{(-)} .
$$

For free quark field, taking $v=(1,0,0,0)$, one has in the momentum space

$$
\begin{aligned}
& \hat{Q}_{v}^{(+)} \rightarrow \frac{1+\psi}{2} u_{s}(p)=\sqrt{\frac{E+m}{2 m}}\left(\begin{array}{c}
1 \\
0
\end{array}\right) \varphi_{s}, \\
& R_{v}^{(+)} \rightarrow \frac{1-\psi}{2} u_{s}(p)=\sqrt{\frac{E+m}{2 m}}\left(\begin{array}{c}
0 \\
\frac{0 \cdot \mathbf{p}}{E+m}
\end{array}\right) \varphi_{s}, \\
& R_{v}^{(-)} \rightarrow \frac{1+\psi}{2} v_{s}(p)=\sqrt{\frac{E+m}{2 m}}\left(\begin{array}{c}
\frac{\sigma \cdot \mathbf{p}}{E+m} \\
0
\end{array}\right) \chi_{s}, \\
& \hat{Q}_{v}^{(-)} \rightarrow \frac{1-\not}{2} v_{s}(p)=\sqrt{\frac{E+m}{2 m}}\left(\begin{array}{c}
0 \\
1
\end{array}\right) \chi_{s} .
\end{aligned}
$$

It is then obvious from Eqs.(2.13)-(2.16) that $\hat{Q}_{v}^{(+)}$and $\hat{Q}_{v}^{(-)}$may be regarded as the "large components" of "quark" and "antiquark" respectively, while $R_{v}^{(+)}$and $R_{v}^{(-)}$are the corresponding "small components" in the case $|\mathbf{p}| \ll E+m$.

Here it should be noted that though the "large" and "small" components of quark and antiquark fields can be understood easily in this way by taking a special vector $v=(1,0,0,0)$, the vector $v$ does not have to be chosen in this way. In fact, rewriting the derivative operator as

$$
\not D=\not D_{1}+\not D_{\perp}
$$

with

$$
\not D_{\mid} \equiv \not v \cdot D, \quad \not D_{\perp} \equiv \not D-\not p(v \cdot D),
$$

and using Eq.(2.3) as well as the commutation relations

$$
\left[\not \varnothing, \not \not_{\|}\right]=0, \quad\left\{\not \varnothing, \not D_{\perp}\right\}=0,
$$

one can easily find the relations between the fields $R_{v}^{( \pm)}\left(\bar{R}_{v}^{( \pm)}\right)$and $\hat{Q}_{v}^{( \pm)}\left(\overline{\hat{Q}}_{v}^{( \pm)}\right)$:

$$
\begin{gathered}
\left(i \not D_{\|}-m_{Q}\right) R_{v}^{( \pm)}+i \not D_{\perp} \hat{Q}_{v}^{( \pm)}=0 \\
\bar{R}_{v}^{( \pm)}\left(-i \overleftarrow{D}_{\|}-m_{Q}\right)-\overline{\hat{Q}}_{v}^{( \pm)} i \overleftarrow{\not D}_{\perp}=0
\end{gathered}
$$

For any operator $O$, the operator $\overleftarrow{O}$ is defined via $\int \kappa \overleftarrow{O} \varphi \equiv-\int \kappa O \varphi$. Thus the fields $Q$ and $\bar{Q}$ can be represented by $\hat{Q}_{v}$ and $\overline{\hat{Q}}_{v}$,

$$
\begin{gathered}
Q=\left[1+\left(1-\frac{i \not v v \cdot D+m_{Q}}{2 m_{Q}}\right)^{-1} \frac{i \not D_{\perp}}{2 m_{Q}}\right] \hat{Q}_{v} \equiv \hat{\omega} \hat{Q}_{v}, \\
\bar{Q}=\overline{\hat{Q}}_{v}\left[1+\frac{-i \overleftarrow{D}_{\perp}}{2 m_{Q}}\left(1-\frac{-i \not p v \cdot \overleftarrow{D}+m_{Q}}{2 m_{Q}}\right)^{-1}\right] \equiv \overline{\hat{Q}}_{v} \overleftarrow{\hat{\omega}}
\end{gathered}
$$

From Eqs.(2.8), (2.9) and (2.22), (2.23) one can see that, in this representation, $R_{v}$ is $1 / m_{Q}$ suppressed compared to $\hat{Q}_{v}$. Therefore $\hat{Q}_{v}^{( \pm)}$and $R_{v}^{( \pm)}$can be regarded as the "large component" and "small component" of the heavy quark field $Q^{( \pm)}$respectively. This is independent of the special choice of vector $v^{\mu}$ as long as it satisfies the condition $v^{2}=1$.

To construct the effective field theory of large component $\hat{Q}_{v}$, one can simply integrate out the small component $R_{v}$, or integrate in the contributions of small component to the effective Lagrangian. This has been shown $[20,21]$ to be equivalent to substitute Eqs.(2.22),(2.23) into the QCD Lagrangian. Thus the resulting large component QCD can be written as 


$$
\mathcal{L}_{Q C D}=\mathcal{L}_{\text {light }}+\mathcal{L}_{Q, v}
$$

with

$$
\begin{aligned}
\mathcal{L}_{Q, v} & \left.\equiv \bar{Q}\left(i \not D-m_{Q}\right) Q\right|_{Q \rightarrow \hat{\omega} \hat{Q}_{v}} \\
& =\overline{\hat{Q}}_{v}\left(i \not p v \cdot D-m_{Q}\right) \hat{Q}_{v}+\frac{1}{2 m_{Q}} \overline{\hat{Q}}_{v} i \not D_{\perp}\left(1-\frac{i \not p v \cdot D+m_{Q}}{2 m_{Q}}\right)^{-1} i \not D_{\perp} \hat{Q}_{v} \\
& +\frac{1}{2 m_{Q}} \overline{\hat{Q}}_{v} i \not D_{\perp}\left(1-\frac{i \not p v \cdot D+m_{Q}}{2 m_{Q}}\right)^{-1}\left(i \not p v \cdot D-m_{Q}\right) \hat{Q}_{v} \\
& +\frac{1}{4 m_{Q}^{2}} \overline{\hat{Q}}_{v}\left(-i \overleftarrow{D}_{\perp}\right)\left(1-\frac{-i \not p v \cdot \overleftarrow{D}+m_{Q}}{2 m_{Q}}\right)^{-1} i \not D_{\perp}\left(1-\frac{i \not p v \cdot D+m_{Q}}{2 m_{Q}}\right)^{-1} i \not D_{\perp} \hat{Q}_{v} .
\end{aligned}
$$

From the definitions (2.10) and the commutation relations (2.19), one can see that there is no quark-antiquark coupling in the first two terms on the rhs. of Eq.(2.25). The last two terms on the rhs. of Eq.(2.25) arise from the quark-antiquark coupling interactions, which has been ignored in the HQET Lagrangian. One can rewrite the above Lagrangian $\mathcal{L}_{Q, v}$ in the following form to see explicitly such features,

$$
\mathcal{L}_{Q, v}=\mathcal{L}_{Q, v}^{(++)}+\mathcal{L}_{Q, v}^{(--)}+\mathcal{L}_{Q, v}^{(+-)}+\mathcal{L}_{Q, v}^{(-+)}
$$

with

$$
\begin{aligned}
\mathcal{L}_{Q, v}^{( \pm \pm)} & =\overline{\hat{Q}}_{v}^{( \pm)}\left[i \hat{\mathcal{P}}_{v}-m_{Q}\right] \hat{Q}_{v}^{( \pm)} \\
\mathcal{L}_{Q, v}^{( \pm)} & =\frac{1}{2 m_{Q}} \overline{\hat{Q}}_{v}^{( \pm)}\left(i \overleftarrow{\mathcal{P}}_{v}-m_{Q}\right)\left(1-\frac{i \not p v \cdot D+m_{Q}}{2 m_{Q}}\right)^{-1}\left(i \not D_{\perp}\right) \hat{Q}_{v}^{(\mp)} \\
& =\frac{1}{2 m_{Q}} \overline{\hat{Q}}_{v}^{( \pm)}\left(-i \overleftarrow{\not}_{\perp}\right)\left(1-\frac{-i \not p v \cdot \overleftarrow{D}+m_{Q}}{2 m_{Q}}\right)^{-1}\left(i \hat{\mathcal{P}}_{v}-m_{Q}\right) \hat{Q}_{v}^{(\mp)}
\end{aligned}
$$

where the operator $i \hat{\mathcal{p}}_{v}$ is defined as

$$
i \hat{\mathcal{P}}_{v}=i \not p v \cdot D+\frac{1}{2 m_{Q}} i \not D_{\perp}\left(1-\frac{i \not v v \cdot D+m_{Q}}{2 m_{Q}}\right)^{-1} i \not D_{\perp} .
$$

The operator $\overleftarrow{\mathcal{P}}_{v}$ in the above equations can be obtained by replacing $D^{\mu}$ with $-\overleftarrow{D^{\mu}}$ in $\hat{\mathcal{P}}_{v}$. Note that the above Lagrangian actually holds for either heavy or light quarks as it is obtained by just integrating out the field component $R_{v}$. For heavy quarks with $|\mathbf{p}| \ll E+m_{Q}$, one can then make expansion in terms of inverse powers of heavy quark mass $m_{Q}$. The resulting effective theory is called HQEFT. It is different from the HQET in which only $\mathcal{L}_{Q, v}^{(++)}$(or $\mathcal{L}_{Q, v}^{(--)}$) in Eq.(2.26) is taken to be the effective Lagrangian. Obviously, the HQET is not complete. In fact, in any physical process of heavy quark, there is heavy quark-antiquark coupling interactions due to the finite mass of heavy quark. Such quark-antiquark coupling terms will provide important contributions at the order of $1 / m_{Q}$. Therefore in order to calculate correctly these $1 / m_{Q}$ corrections arising from the finite mass of heavy quark, one should take into account the contributions from the terms $\mathcal{L}_{Q, v}^{( \pm \mp)}$ and $\mathcal{L}_{Q, v}^{(--)}$for the processes involving the heavy quark $Q_{v}^{(+)}$or from the terms $\mathcal{L}_{Q, v}^{( \pm \mp)}$ and $\mathcal{L}_{Q, v}^{(++)}$ for the ones involving the heavy antiquark $Q_{v}^{(-)}$.

For making $1 / m_{Q}$ expansion, it is useful to remove the large mass term in the above Lagrangian. We then introduce new field variables $Q_{v}$ and $\bar{Q}_{v}$ with the definition

$$
Q_{v}=e^{i \not m_{Q} v \cdot x} \hat{Q}_{v}, \quad \bar{Q}_{v}=\overline{\hat{Q}}_{v} e^{-i \not m_{Q} v \cdot x} .
$$

Noticing the feature that $\not \varnothing$ commutes with $\not_{\|}$but anticommutes with $\not_{\perp}$, we can rewrite above Lagrangian to be 


$$
\begin{aligned}
\mathcal{L}_{Q, v}^{( \pm \pm)} & =\bar{Q}_{v} i \mathcal{P}_{v} Q_{v} \\
\mathcal{L}_{Q, v}^{( \pm \mp)} & =\frac{1}{2 m_{Q}} \bar{Q}_{v}\left(i \overline{\mathcal{D}}_{v}\right) e^{2 i \not m_{Q} v \cdot x}\left(1-\frac{i \not p v \cdot D}{2 m_{Q}}\right)^{-1}\left(i \not D_{\perp}\right) Q_{v} \\
& =\frac{1}{2 m_{Q}} \bar{Q}_{v}\left(-i \overleftarrow{D}_{\perp}\right)\left(1-\frac{-i \not p v \cdot \overleftarrow{D}}{2 m_{Q}}\right)^{-1} e^{-2 i \not m_{Q} v \cdot x}\left(i \mathcal{D}_{v}\right) Q_{v}
\end{aligned}
$$

with

$$
\begin{aligned}
& i \not_{v}=i \not b v \cdot D+\frac{1}{2 m_{Q}} i D_{\perp}\left(1-\frac{i \not p v \cdot D}{2 m_{Q}}\right)^{-1} i \not D_{\perp} \\
& i \overline{\mathcal{D}}_{v}=-i \not b v \cdot \overleftarrow{D}+\frac{1}{2 m_{Q}}\left(-i \overleftarrow{D}_{\perp}\right)\left(1-\frac{-i \not p v \cdot \overleftarrow{D}}{2 m_{Q}}\right)^{-1}\left(-i \overleftarrow{D}_{\perp}\right)
\end{aligned}
$$

where we have expressed the effective Lagrangian for quark-antiquark coupling interactions (2.32) in terms of two identical formulations. One can use either of them depending on the convenience for the relevant applications.

The above Lagrangian (eqs.(2.26-29) or eqs.(2.31-33)) forms the basic framework of HQEFT which has first been derived in ref. [3]. Here we rewrite it in a more compact expression. Note that the resulting effective Lagrangian is exact for the quark component $Q_{v}=Q_{v}^{(+)}+Q_{v}^{(-)}$. What we have done is making the field redefinitions and integrating out the quark field component $R_{v}=R_{v}^{(+)}+R_{v}^{(-)}$, or integrating in the contributions of the quark component $R_{v}=R_{v}^{(+)}+R_{v}^{(-)}$ to the effective Lagrangian. It then becomes manifest why we shall base on the HQEFT of QCD rather than the HQET.

\section{EFFECTS OF QUARK-ANTIQUARK COUPLING TERMS AND CONSISTENCY OF HQEFT}

The Lagrangian (2.26) contains the fields of quark and antiquark explicitly. It is then natural to ask what is the new effects of HQEFT with the quark-antiquark coupling terms. For that, it is better to consider the concrete physical processes. But for many physical processes, the initial and final states contain no antiquark (or quark), and therefore in these cases it is convenient to deal with only the effective quark (or antiquark) field variables. From this consideration it is useful to further derive a Lagrangian represented only by quark (or antiquark) field variables but effectively taking the contributions of quark-antiquark coupling terms into account.

From the Lagrangian presented in Eqs.(2.26)-(2.28) it is easy to get the relations between quark and antiquark field variables:

$$
\begin{aligned}
& \left(\hat{\mathcal{P}}_{v}-m_{Q}\right) \hat{Q}_{v}^{(-)}+\left[i \not D_{\perp}\left(m_{Q}-i \not b v \cdot D\right)^{-1}\left(i \hat{\mathcal{P}}_{v}-m_{Q}\right)\right] \hat{Q}_{v}^{(+)}=0 \\
& \overline{\hat{Q}}_{v}^{(-)}\left(\overleftarrow{\mathcal{P}}_{v}-m_{Q}\right)+\overline{\hat{Q}}_{v}^{(+)}\left[i \overleftarrow{\not}_{\perp}\left(m_{Q}-i \not b v \cdot \overleftarrow{D}\right)^{-1}\left(i \overleftarrow{\mathcal{P}}_{v}-m_{Q}\right)\right]=0
\end{aligned}
$$

or

$$
\begin{aligned}
& \hat{Q}_{v}^{(-)}=-\left(\hat{\mathcal{P}}_{v}-m_{Q}\right)^{-1}\left[i \not D_{\perp}\left(m_{Q}-i \not p v \cdot D\right)^{-1}\left(i \hat{\mathcal{P}}_{v}-m_{Q}\right)\right] \hat{Q}_{v}^{(+)} \\
& \overline{\hat{Q}}_{v}^{(-)}=-\overline{\hat{Q}}_{v}^{(+)}\left[i \overleftarrow{\mathcal{D}}_{\perp}\left(m_{Q}-i \not p v \cdot \overleftarrow{D}\right)^{-1}\left(i \overleftarrow{\mathcal{P}}_{v}-m_{Q}\right)\right]\left(\overleftarrow{\mathcal{P}}_{v}-m_{Q}\right)^{-1}
\end{aligned}
$$

Integrating out the heavy antiquark fields is equivalent to substituting Eqs.(3.3), (3.4) into Eqs.(2.26)-(2.28). Consequently, we arrive at the effective Lagrangian

$$
\mathcal{L}_{\text {eff }}^{(++)}=\mathcal{L}_{Q, v}^{(++)}+\tilde{\mathcal{L}}_{Q, v}^{(++)},
$$

which is nothing but representing the Lagrangian (2.26) in terms of only quark variables. The second part $\tilde{\mathcal{L}}_{Q, v}^{(++)}$comes from the contributions of integrating out antiquark. Its explicit form is found to be 


$$
\begin{aligned}
\tilde{\mathcal{L}}_{Q, v}^{(++)} & =\left.\left\langle\mathcal{L}_{Q, v}^{(--)}+\mathcal{L}_{Q, v}^{(+-)}+\mathcal{L}_{Q, v}^{(-+)}\right\rangle\right|_{\hat{Q}_{v}^{(-)} \rightarrow \hat{Q}_{v}^{(+)}} \\
& =-\overline{\hat{Q}}_{v}^{(+)} i \not D_{\perp}\left(m_{Q}-i \not p v \cdot D\right)^{-1} i \not D_{\perp}\left(m_{Q}-i \not \not v \cdot D\right)^{-1}\left(i \hat{\mathcal{P}}_{v}-m_{Q}\right) \hat{Q}_{v}^{(+)} .
\end{aligned}
$$

After removing the large mass term in the above effective Lagrangian, we then obtain the following compact form

$$
\mathcal{L}_{\text {eff }}^{(++)}=\bar{Q}_{v}^{(+)} i \mathcal{D}_{v} \frac{1}{i \not p v \cdot D} i \mathcal{D}_{v} Q_{v}^{(+)}
$$

Note that up to now we do not yet make any approximation except making redefinitions for the quark fields and integrating out the field component $R_{v}$ and antiquark field $\hat{Q}_{v}^{(-)}$. So that the above effective Lagrangian should still characterize the necessary features of QCD.

To be manifest, we may reexpress the above Lagrangian in the following explicit form

$$
\begin{aligned}
\mathcal{L}_{\text {eff }}^{(++)}= & \bar{Q}_{v}^{(+)}\left\{i \not b v \cdot D+2 \cdot \frac{1}{2 m_{Q}}\left(i \not D_{\perp}\right)\left(1-\frac{i \not p v \cdot D}{2 m_{Q}}\right)^{-1}\left(i \not D_{\perp}\right)\right\} Q_{v}^{(+)} \\
& +\frac{1}{4 m_{Q}^{2}} \bar{Q}_{v}^{(+)}\left(i \not D_{\perp}\right)\left(1-\frac{i \not b v \cdot D}{2 m_{Q}}\right)^{-1}\left(i \not D_{\perp}\right) \\
& \times \frac{1}{i \not b v \cdot D}\left(i \not D_{\perp}\right)\left(1-\frac{i \not p v \cdot D}{2 m_{Q}}\right)^{-1}\left(i \not D_{\perp}\right) Q_{v}^{(+)} .
\end{aligned}
$$

It is easily seen that the above effective Lagrangian for quark fields is distinguishable from the effective Lagrangian adopted in the HQET. There are actually two additional terms which arise from the contributions by integrating out the antiquark fields. One term is at $1 / m_{Q}$ order, it exactly coincides with the one in $\mathcal{L}_{Q, v}^{(+)}$. This explains the factor of two in the second term of the curl bracket in the above effective Lagrangian. The other term is formally at $1 / m_{Q}^{2}$ order but with the operator $\not v v \cdot D$ in the denominator, which has to be treated carefully for different physical processes when making $1 / m_{Q}$ expansion. Their physical consequences will be discussed in detail below. Such two additional terms explicitly represent new effects of HQEFT vs. HQET.

Before proceeding, we shall first check the consistency of the above HQEFT. Since the following issue naturally arises: as the above Lagrangian is exact without making any approximations, it should also recover the case for the free quark fields. The question is then how to understand the additional two terms formally at order $1 / m_{Q}$ and $1 / m_{Q}^{2}$. The answer is positive as one can see below.

For the free quark case, there is no coupling between quark and gluon. One can simply take the coupling constant to be zero and make the replacement

$$
\begin{aligned}
& D_{\|}^{\mu} \rightarrow \partial_{\|}^{\mu}=v^{\mu} v \cdot \partial \\
& D_{\perp}^{\mu} \rightarrow \partial_{\perp}^{\mu}=\partial^{\mu}-v^{\mu} v \cdot \partial, \\
& \mathcal{P}_{v} \rightarrow i \not_{v}=i \not \partial_{\|}+\frac{1}{2 m_{Q}} i \not_{\perp}^{2}\left(1+\frac{i \not_{\|}}{2 m_{Q}}\right)^{-1} .
\end{aligned}
$$

Thus the Lagrangian for the free quark field is simply obtained from Eqs.(3.7)-(3.9),

$$
\begin{aligned}
\mathcal{L}_{\text {eff }}^{(++)}= & \bar{Q}_{v}^{(+)} i \not \partial_{v} \frac{1}{i \not_{\|}} i \not_{v} Q_{v}^{(+)} \\
= & \bar{Q}_{v}^{(+)}\left\{i \not \not_{\|}+2 \cdot \frac{1}{2 m_{Q}}\left(i \not \not_{\perp}\right)^{2}\left(1+\frac{i \not_{\|}}{2 m_{Q}}\right)^{-1}\right\} Q_{v}^{(+)} \\
& +\frac{1}{4 m_{Q}^{2}} \bar{Q}_{v}^{(+)}\left(i \not_{\perp}\right)^{4}\left(1+\frac{i \not_{\|}}{2 m_{Q}}\right)^{-2} \frac{1}{i \not_{\|}} Q_{v}^{(+)}
\end{aligned}
$$

Notice the factor of two at the $1 / m_{Q}$ order in the first term and also the denominator $i \not_{\|}$in the second term which is formally at $1 / m_{Q}^{2}$ order. It appears to be quite unusual. We shall see that 
the second term is not truly at the order of $1 / m_{Q}^{2}$ for the free quark field due to the denominator $i \not \phi_{\|}$. Its leading contribution is actually at $1 / m_{Q}$ order with magnitude being half to the $1 / m_{Q}$ order part in the first term but with opposite sign.

To be convenient, let us turn to the momentum space, $i \partial_{v}$ is represented as

$$
v \cdot k+\frac{k^{2}-(v \cdot k)^{2}}{2 m_{Q}}\left(1+\frac{v \cdot k}{2 m_{Q}}\right)^{-1} .
$$

Note that when taking $v=(1,0,0,0)$, it is simplified to be

$$
k^{0}-\frac{\mathbf{k}^{2}}{2 m_{Q}}\left(1+\frac{k^{0}}{2 m_{Q}}\right)^{-1}
$$

As the free quark is on mass-shell, taking $v=(1,0,0,0)$ for simplicity, one has

$$
\begin{aligned}
& P^{2}=\left(m_{Q} v+k\right)^{2}=m_{Q}^{2}, \rightarrow k^{0}-\frac{\mathbf{k}^{2}}{2 m_{Q}}\left(1+\frac{k^{0}}{2 m_{Q}}\right)^{-1}=0 \\
& \text { or } \quad k_{0}=\sqrt{m_{Q}^{2}+\mathbf{k}^{2}}-m_{Q}=\frac{\mathbf{k}^{2}}{2 m_{Q}}+O\left(\frac{1}{m_{Q}^{2}}\right) .
\end{aligned}
$$

In the coordinate space, Eq.(3.11) can be represented in the form of operators as

$$
i \not \partial_{\|}=-\frac{1}{2 m_{Q}} i \not \not_{\perp}^{2}\left(1+\frac{i \not \partial_{\|}}{2 m_{Q}}\right)^{-1}=-\frac{\left(i \not \partial_{\perp}\right)^{2}}{2 m_{Q}}+O\left(\frac{1}{m_{Q}^{2}}\right) .
$$

Relation (3.12) means that when the quark is on mass-shell, the longitudinal and transverse momenta are not at the same order in the $1 / m_{Q}$ expansion. In fact, the operator $i \not \not_{\|}$should be treated at the order of $1 / m_{Q}$. More exactly it is at the same order of $\left(i \not \not_{\perp}\right)^{2} / 2 m_{Q}$.

Substituting the relation (3.12) into the second term of the Lagrangian in Eq.(3.10), one has

$$
\begin{aligned}
\mathcal{L}_{\text {eff }}^{(++)} & =\bar{Q}_{v}^{(+)}\left\{i \not_{\|}+\frac{1}{m_{Q}}\left(i \partial_{\perp}\right)^{2}\left(1+\frac{i \not_{\|}}{2 m_{Q}}\right)^{-1}\right\} Q_{v}^{(+)} \\
& -\frac{1}{4 m_{Q}^{2}} \bar{Q}_{v}^{(+)}\left(i \not_{\perp}\right)^{4}\left(1+\frac{i \not_{\|}}{2 m_{Q}}\right)^{-1}\left[\frac{\left.i \not_{\perp}\right)^{2}}{2 m_{Q}}\right]^{-1} Q_{v}^{(+)} \\
& =\bar{Q}_{v}^{(+)}\left\{i \not_{\|}+\frac{1}{2 m_{Q}} i \not_{\perp}^{2}\left(1+\frac{i \not_{\|}}{2 m_{Q}}\right)^{-1}\right\} Q_{v}^{(+)} .
\end{aligned}
$$

It is seen that the resulting Lagrangian (3.13) is self-consistent with the on mass-shell condition (3.11) or (3.12). This becomes the known Lagrangian for a free quark field. One then arrives at a consistent check for HQEFT in the free quark case.

\section{HQEFT AS A LARGE COMPONENT QCD OF HEAVY QUARKS}

We now apply the exact formulation of HQEFT to the heavy quark systems with $p \ll m_{Q}$. For this case, one may expand the HQEFT Lagrangian (3.8) into a series in powers of the inverse heavy quark mass. Practically, in studying certain physical processes it is reliable to consider only some lower order contributions in the Lagrangian but neglect the higher order $1 / m_{Q}$ corrections. In order to correctly perform this expansion, however, one must keep an eye on the physical conditions related to the concrete processes. In particular, the longitudinal and transverse momenta of the heavy quark may be at different powers for different physical processes. Correspondingly, the operator $\not_{\|}$and $\not_{\perp}$ may be treated as operators in different powers in the expansion. To be more explicit, we consider two interesting physical systems which are corresponding to two typical conditions. 
We first consider the heavy quark within heavy quarkonia systems, which is known to be well treated by NRQCD. In fact, such a heavy quark is considered to be nearly on mass-shell. This implies the operator relation for the $1 / m_{Q}$ expansion

$$
i \not b v \cdot D=-\frac{\left(i D_{\perp}\right)^{2}}{2 m_{Q}}+O\left(\frac{1}{m_{Q}^{2}}\right) .
$$

With this condition, it is not difficult to show that the effective Lagrangian (Eq.(3.8)) has the following form in terms of the $1 / m_{Q}$ expansion

$$
\mathcal{L}_{\text {eff }}^{(++)}=\bar{Q}_{v}^{(+)}\left\{i \not b v \cdot D+\frac{1}{2 m_{Q}}\left(i \not D_{\perp}\right)^{2}\right\} Q_{v}^{(+)}+O\left(\frac{1}{m_{Q}^{2}}\right) .
$$

Taking $v=(1,0,0,0)$, one has

$$
\mathcal{L}_{\text {eff }}^{(++)}=\bar{Q}_{v}^{(+)}\left\{i \frac{\partial}{\partial t}+\frac{\nabla^{2}}{2 m_{Q}}\right\} Q_{v}^{(+)}+O\left(\frac{1}{m_{Q}^{2}}\right) .
$$

which does result in an NRQCD.

We now consider another interesting case with heavy quark being slightly off mass-shell. This is considered to be the case for a single heavy quark within the heavy hadron. The magnitude of the off mass-shell is usually assumed to be at the order of bounding energy. Namely it satisfies the off shell condition

$$
\frac{P^{2}-m_{Q}^{2}}{2 m_{Q}} \sim \bar{\Lambda}
$$

Taking $P=m_{Q} v+k$ with $v=(1,0,0,0)$, one has

$$
k^{0} \sim|\mathbf{k}| \sim \bar{\Lambda},
$$

which has been considered in both HQET and HQEFT. $\bar{\Lambda} \sim 500 \mathrm{MeV}$ is the typical bounding energy of heavy hadrons. In general, one may take the corresponding approximation in the operator form:

$$
\left\langle i \not D_{\|}\right\rangle \sim\left\langle i \not D_{\perp}\right\rangle \sim \bar{\Lambda},
$$

which implies that the operators corresponding to longitudinal and transverse momenta are taken to be at the same order in the $1 / m_{Q}$ expansion.

With the above considerations, the Lagrangian (3.8) can be expanded in terms of $1 / m_{Q}$ into the following form

$$
\mathcal{L}_{\text {eff }}^{(++)}=\mathcal{L}_{\text {eff }}^{(0)}+\mathcal{L}_{\text {eff }}^{\left(1 / m_{Q}\right)}
$$

with

$$
\begin{aligned}
\mathcal{L}_{\text {eff }}^{(0)}= & \bar{Q}_{v}^{(+)}(i \not p v \cdot D) Q_{v}^{(+)} \\
\mathcal{L}_{\text {eff }}^{\left(1 / m_{Q}\right)}= & 2 \frac{1}{2 m_{Q}} \bar{Q}_{v}^{(+)}\left(i \not D_{\perp}\right)^{2} Q_{v}^{(+)}+2 \frac{1}{4 m_{Q}^{2}} \bar{Q}_{v}^{(+)} i \not D_{\perp}(i \not \gamma v \cdot D) i \not D_{\perp} Q_{v}^{(+)} \\
& +\frac{1}{4 m_{Q}^{2}} \bar{Q}_{v}^{(+)}\left(i \not D_{\perp}\right)^{2} \frac{1}{i \not v v \cdot D}\left(i \not D_{\perp}\right)^{2} Q_{v}^{(+)}+O\left(\frac{1}{m_{Q}^{3}}\right)
\end{aligned}
$$

where $\mathcal{L}_{\text {eff }}^{(0)}$ is the leading term and possesses the spin-flavor symmetry. The second part $\mathcal{L}_{\text {eff }}^{\left(1 / m_{Q}\right)}$ contains the remaining terms which are the spin-flavor symmetry breaking ones but suppressed by $1 / m_{Q}$. Note that the present case differs from the first case of the nearly on mass-shell heavy quark. The third term in the rhs. of Eq.(4.7) is now regarded as at the order of $1 / m_{Q}^{2}$. So the first 
term in the rhs. of Eq.(4.7) is now regarded as the total $1 / m_{Q}$ order correction to the leading order Lagrangian (4.6). Obviously, the factor of two in this case can be understood easily. It makes the HQEFT different from the HQET when applying to the physical systems in which the single heavy quark within a hadron is slightly off mass-shell, i.e., $\left\langle i \not D_{\mid}\right\rangle \sim\left\langle i D_{\perp}\right\rangle \sim \bar{\Lambda}$. Their physical effects will be discussed below.

The above analysis shows that HQEFT provides a simple and systematic framework when the physical processes allow to make the expansions in terms of the inverse powers of heavy quark mass $m_{Q}$. In this sense, the HQEFT is nothing but a large component QCD of heavy quarks.

\section{NEW FEATURES OF HQEFT VS. HQET}

The quark-antiquark coupling terms in HQEFT should exhibit their effects in its phenomenological applications to physical processes. Some detailed analyses have been made in references [20]- [29]. Here we revisit the most important features of HQEFT with paying attention to the comparison with HQET. In general, HQEFT will lead to the results deviating from those in HQET starting at $1 / m_{Q}$ order. Especially, the HQEFT can largely simplify the evaluations of hadronic matrix elements in comparison with the HQET.

First of all, a straightforward and automatic consequence in HQEFT of QCD is that at zero recoil point the weak transition matrix elements between ground state heavy mesons do not receive $1 / m_{Q}$ order corrections. Of course this was known to the so-called Luke's theorem in HQET. However, in HQET one has to make a carefully proof with the aid of the equation of motion $i v \cdot D Q_{v}^{(+)}=0$ to arrive at this statement. Also HQEFT of QCD is automatically reparametrization invariant [3] as it is derived directly from QCD, whereas in HQET one needs to impose certain conditions in order to insure the reparametrization invariance [35,36].

The HQEFT of QCD displays its advantage in the applications to the extraction of the important CKM matrix element $\left|V_{c b}\right|$. It is known that $\left|V_{c b}\right|$ can be extracted by studying $B \rightarrow D^{*} l \nu$ and $B \rightarrow D l \nu$ decays. In HQET the latter decay channel $B \rightarrow D l \nu$ provides a less precise one in determining $\left|V_{c b}\right|$. Besides the experimental reasons, an important reason for this is that the Luke's theorem in HQET no longer suppresses the $1 / m_{Q}$ corrections for the $B \rightarrow D l \nu$ decay width. This may induce large theoretical uncertainty to the extracted $\left|V_{c b}\right|$. The situation becomes quite different in the HQEFT of QCD, where the suppression of $1 / m_{Q}$ corrections is valid also for the $B \rightarrow D l \nu$ channel. As a consequence, HQEFT of QCD provides a more precise way than HQET to extract $\left|V_{c b}\right|$.

It is noted that in HQEFT the $1 / m_{Q}$ corrections to heavy meson masses can be related to the $1 / m_{Q}$ corrections to weak transition matrix elements between the relevant heavy mesons. While in HQET these are treated separately and characterized by different sets of functions (factors).

It is also very interesting to observe that heavy hadron dynamics is much simplified in HQEFT than in HQET. Specifically, fewer independent functions (factors) are needed in HQEFT than in HQET to characterize the finite mass corrections. For instance, in HQET one needs 6 wave functions $\xi_{i}$ and $\chi_{i}(i=1,2,3)$ to parametrize the $1 / m_{Q}$ order corrections to weak transition matrix elements between heavy mesons, and needs other 2 factors $\lambda_{1}$ and $\lambda_{2}$ for the $1 / m_{Q}$ order corrections to meson masses. In HQEFT the $1 / m_{Q}$ corrections from current expansion and from insertion of $1 / m_{Q}$ order Lagrangian are attributed to the same set of 3 wave functions $\kappa_{i}(i=1,2,3)$; and the $1 / m_{Q}$ order corrections to meson masses are also naturally related to the zero recoil values of $\kappa_{1}$ and $\kappa_{2}$. Instead of the 8 functions (factors) needed in HQET, one thus needs only 3 independent functions in HQEFT.

In studying inclusive bottom hadron decays, the mass quantity entering into decay rates in the HQEFT formulation is no longer the heavy quark mass $m_{Q}$ but the "dressed heavy quark" mass $\hat{m}_{Q}=m_{Q}+\bar{\Lambda}$, which is related to the hadron mass at a high order $1 / m_{Q}$ corrections, i.e., $\hat{m}_{Q}=m_{H}\left[1+O\left(1 / m_{Q}^{2}\right)\right]$. In this way the inclusive decay rate formula of heavy hadrons is found to have no $1 / \hat{m}_{Q}$ order correction when it is given by the physical heavy hadron mass. This is because the HQEFT of QCD allows us to perform the heavy quark expansion at the point of the "dressed heavy quark" mass $\hat{m}_{Q}$ instead of $m_{Q}$, which successfully suppresses the next to leading order contributions of the expansion. Consequently, the HQEFT of QCD provides a precise 
determination of $\left|V_{c b}\right|$ and $\left|V_{u b}\right|$ in the inclusive bottom hadron decays. In particular, it can also present a well explanation for the puzzle of the bottom hadron life time differences, i,e., the ratios $\tau\left(B_{s}^{0}\right) / \tau\left(B^{0}\right)$ and $\tau\left(\Lambda_{b}\right) / \tau\left(B^{0}\right)$.

In the above we briefly describe only some of the most important features of HQEFT as compared to HQET. Actually, HQEFT of QCD has also been applied to study the issues such as the charm counting in the bottom hadron systems, the heavy meson decay constants, heavy-to-light meson decays and so on. These applications have shown the power of HQEFT for its consistency. All of them are attributed to the correct treatment for the quark-antiquark coupling terms. For more detailed discussions on these and other features, we refer to the refs. [20]- [29]. Note that the above interesting features of HQEFT cannot be obtained from the usual HQET through a simple redefinition for the heavy quark fields.

In conclusion, HQEFT is nothing but a large component QCD of heavy quarks. Its consistency and completeness become manifest, which strongly suggests that we shall base on the HQEFT of QCD rather than the usual HQET to investigate heavy quark systems beyond the leading order of $1 / m_{Q}$ expansion. We hope that the interesting features and consistent results concerning the phenomenological applications of HQEFT will invoke more and more attention to this effective theory.

\section{Acknowledgement}

The authors (WYW and YLW) would like to thank K. Hagiwara for stimulating discussions and conversation. They are also grateful to Y.Q. Chen, Y.B. Dai, C.S. Huang, T. Huang, X.Y. Li, Y.P. Kuang and many colleagues for useful discussions. This work was supported in part by the key projects of Chinese Academy of Sciences and National Science Foundation of China.

[1] H. Georgi, Phys. Lett. B 240 (1990) 447.

[2] For review see e.g., M. Neubert, Phys. Rept. 245 (1994) 259.

[3] Y. L. Wu, Mod. Phys. Lett. A 8 (1993) 819.

[4] E. V. Shuryak, Phys. Lett. B 93 (1980) 134; Nucl. Phys. B 198 (1982) 83.

[5] S. Nussinov, W. Wetzel, Phys. Rev. D 36 (1987) 130.

[6] N. Isgur, M. Wise, Phys. Lett. B 232 (1989) 113; B 237 (1990) 527; B 206 (1988) 681.

[7] M. B. Voloshin, M. A. Shifman, Sov. J. Nucl. Phys. 45 (1987) 292; 47 (1988) 199.

[8] E. Eichten, B. Hill, Phys. Lett. B 234 (1990) 511; E. Eichten, Nucl. Phys. Proc. Suppl. B 4 (1988) 170.

[9] B. Grinstein, Nucl. Phys. B 339 (1990) 253.

[10] A. Falk, H. Georgi, B. Grinstein, M. B. Wise, Nucl. Phys. B 343 (1990) 1.

[11] T. Mannel, Z. Ryzak, Phys. Lett. B 247 (1990) 2388.

[12] F. Hussain, J. G. Körner, K. Schilcher, G. Thompson, Y. L. Wu, Phys. Lett. B 249 (1990) 295.

[13] M. Luke, Phys. Lett. B 252 (1990) 447.

[14] J. G. Körner, G. Thompson, Phys. Lett. B 264 (1991) 185.

[15] Y. L. Wu, Mod. Phys. Lett. A 6 (1991) 1277.

[16] A. F. Falk, B. Grinstein, M. E. Luke, Nucl. Phys. B 357 (1991) 185.

[17] T. Mannel, W. Roberts, Z. Ryzak, Nucl. Phys. B 368 (1992) 204.

[18] Y.B. Dai, C.S. Huang, H.Y. Jin, Z.Phys. C60 527 (1993).

[19] M. B. Wise, 'Heavy Quark Physics', CALT-68-2172, hep-ph/9805468.

[20] W. Y. Wang, Y. L. Wu, Y. A. Yan, Int. J. Mod. Phys. A 15 (2000) 1817.

[21] Y. A. Yan, Y. L. Wu, W. Y. Wang, Int. J. Mod. Phys. A 15 (2000) 2735.

[22] W. Y. Wang, Y. L. Wu, Int. J. Mod. Phys. A 16 (2001) 377.

[23] Y. L. Wu, Y. A. Yan, Int. J. Mod. Phys. A 16 (2001) 285.

[24] W. Y. Wang, Y. L. Wu, Phys. Lett. B 515 (2001) 57.

[25] W. Y. Wang, Y. L. Wu, Phys. Lett. B 519 (2001) 219. 
[26] W. Y. Wang, Y. L. Wu, M. Zhong, to be published in Phys. Rev. D, 2003, hep-ph/0205157.

[27] M. Zhong, Y. L. Wu, W. Y. Wang, to be published in Int. J. Mod. Phys. A, 2003, hep-ph/0206013.

[28] W. Y. Wang, Ph. D. thesis, 2001.

[29] Y. A. Yan, Ph. D. thesis, 2002.

[30] F. Berto, M. A. Sanchis-Lozano, Nuov. Cim. A 112 (1999) 1181.

[31] F. Berto, M.A. Sanchis-Lozano, Nucl. Phys. Proc. Suppl. 93 (2001) 212.

[32] M. A. Sanchis-Lozano, Int. J. Mod. Phys. A 16 (2001) 4189.

[33] W. E. Caswell, G. P. Lepage, Phys. Lett. 167 B (1986) 437.

[34] G. T. Bodwin, E. Braaten, G. P. Lepage, Phys. Rev. D 51 (1995) 1125; Erratum-ibid. Phys. Rev. D 55 (1997) 5853.

[35] M. Neubert, Phys. Lett. B306 357 (1993).

[36] Y.Q. Chen, Phys.Lett. B317 421 (1993). 